\title{
Volterra's kernels-based finite-time parameters estimation of the Chua system
}

\author{
Giuseppe Fedele $^{\mathrm{a}}$, Luigi D'Alfonso ${ }^{\mathrm{b}}$, Gilberto Pin ${ }^{\mathrm{c}}$, Thomas Parisini ${ }^{\mathrm{d}}$ \\ ${ }^{a}$ DIMES, University of Calabria, Italy \\ ${ }^{b}$ GiPStech s.r.l, Italy \\ ${ }^{c}$ Electrolux Italia S.p.A., Italy \\ ${ }^{d}$ Dept. of Electrical and Electronic Engineering, Imperial College London, UK
}

\begin{abstract}
In this work, the unknown set of parameters of the Chua system is recovered under the hypothesis that the voltages of the capacitors are available. The system is shown to be algebraically observable and identifiable with respect to the well chosen outputs. Focusing on the differential equations, the Volterra kernel-based approach is used to perform an estimation without the uncertainty of the unmeasurable derivatives and the unknown initial conditions.
\end{abstract}

Keywords: Chua system, Volterra's kernels, Sliding mode control, Finite-time estimation

\section{Introduction}

Chaotic systems have attracted the attention of the recent research due to their potential applications in secure communication, laser system, electronic chemistry, neurophysiology and ecology [1]. Estimating some variables and unknown parameters of a chaotic system are common and interesting problems in chaos theory and its applications. Aiming at performing a given task or achieving defined performance, all the parameters of a dynamical system have to be known. Moreover, depending on the desired goal, system variables which cannot be measured may be required. In this context, the reconstruction problem consists in extracting some physical parameters simultaneously estimating some non available states, starting from the available system outputs [2].

To solve this problem, methodologies based on control ideas, like state observers design and system identification, have been deployed as mechanisms to obtain the non-available variables $[3,4]$ avoiding the use of expensive resources. Other authors propose the use of delay embedding methods based solutions $[5,6,7,8,9]$. In $[10]$ an approach to provide an accurate estimation of the parameters of a Lorenz system synchronized by an external driving signal is proposed. In particular, the estimation is obtained by translating the Lorenz equation into a linear one w.r.t. the parameters and by obtaining them as a solution of the resulting final equations.

In [11], an approach to estimate the parameters of chaotic systems by properly modifying a noise-induced synchronization is proposed. First of all, a

Email address: giuseppe.fedele@unical.it (Giuseppe Fedele) 
dimensionally-expanded parameter estimating system is designed; the chaotic transmitted signal is then used to synchronize the parameter estimating system and the original chaotic one. Parameters are finally obtained using the synchronized estimating system. Following the same lines, in the last two decades researchers have been attracted by synchronization and identification of chaotic systems [12] especially because when the synchronization of two coupled chaotic systems is achieved, no matter what the initial conditions are, the difference between the systems goes to zero as time goes to infinity $[9,13,14]$. The above property has been used in engineering communications to encode and decode messages $[15,16]$.

When the state of the system is not fully measurable, then the identification problem becomes more difficult due to the necessity to estimate, besides the parameters, also the hidden state variables from the sole input/output measurements. To solve this problem, in [17] an efficient approach for the estimation of the non-available state variables for the Duffings chaotic system is proposed by obtaining an integral output parametrization of the hidden state variables.

Among the Chaotic systems, the Chua circuit has been studied in various ways. The unknown set of parameters of this system has been obtained by [18] using an adaptive control approach and assuming that the voltages of the capacitors are available. In [6], an estimation strategy based on the embedding approach using time delayed outputs has been proposed. In [19], the least squares method has been used to solve the estimation problem, under the strong assumption of all the Chua system states availability. A solution based on a non-asymptotic linear estimator has been used in [20].

In the parameters reconstruction context, properties of Volterra integral operator can be used to obtain useful estimation algorithms able to provide parameters value in finite-time. The kernel-based method designs a class of Bivariate Feedthrough Non-asymptotic Kernels (BF-NK) to form the Volterra integral operator, providing a finite-time parameter estimation for continuous-time linear systems, annihilating the effects of the unknown initial conditions and avoiding the need for output derivatives computations. The Volterra integral operators properties have been widely used in various contexts for the finite time parameters estimation $[21,22,23]$. In this paper, the Chua system is proven to be algebraically observable and identifiable, with respect to the voltages outputs, and starting from the ideas proposed by [23], the Volterra operator with BFNK is used to achieve a joint parametric estimation of the system parameters with arbitrary non-asymptotic convergence properties. It represents a revised version of [24], aiming to provide new contributions in terms of: clarification on the advantages of the proposed strategy, detailed description of the parameters estimation method based on kernels functions and finally presentation of new simulated results. Some comparisons are reported in terms of accuracy and robustness with an existing method in literature. The paper is organized as follows: Section 2 describes the Chua system and its main properties; Section 3 summarizes some of the properties of the Volterra integral operator; in Section 4 the proposed finite time parameters estimation is obtained; numerical simulations proving the effectiveness of the Volterra based estimation are shown in Section 5; finally, Section 6 is devoted to the conclusions.

\section{The Chua system}




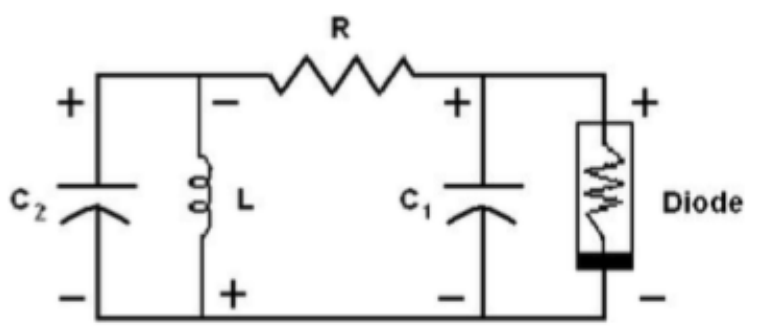

Figure 1: The Chua system.

Chua's circuit in Fig. 1 is the simplest and most widely studied real nonlinear dynamical systems [25]. It consists of three energy-store elements (an inductor and two capacitors), a linear resistor and a single nonlinear resistor, called Chua's diode. A simplified nonlinear model of this system is given by:

$$
\begin{aligned}
C_{1} \frac{d v_{C 1}}{d t} & =\frac{1}{R}\left(v_{C 2}-v_{C 1}\right)-\phi\left(v_{C 1}\right), \\
C_{2} \frac{d v_{C 2}}{d t} & =\frac{1}{R}\left(v_{C 1}-v_{C 2}\right)+i_{L}, \\
L \frac{d i_{L}}{d t} & =-v_{C 2}
\end{aligned}
$$

where $R$ is a linear resistance, $v_{C 1}$ and $v_{C 2}$ are the voltages across capacitors $C_{1}$ and $C_{2}$, respectively, $i_{L}$ is the current through the inductor $L$ and $\phi\left(v_{C 1}\right)$ is the current through the nonlinear resistor as a function of the voltage across capacitor $C_{1}$.

This nonlinear function is described by the odd-symmetric piecewise-linear function

$$
\phi(x)=-\left[m_{1} v_{C 1}+\frac{m_{0}-m_{1}}{2}\left(\left|v_{C 1}+B_{p}\right|-\left|v_{C 1}-B_{p}\right|\right)\right],
$$

where $m_{0}, m_{1}$ and $B_{p}$ are three fixed constants of the diode. The system (1) can be rewritten in dimensionless form as

$$
\begin{aligned}
& \dot{x}_{1}=\beta\left(-x_{1}+x_{2}-f\left(x_{1}\right)\right) \\
& \dot{x}_{2}=x_{1}-x_{2}+x_{3} \\
& \dot{x}_{3}=-\gamma x_{2}
\end{aligned}
$$

with

$$
f(x)=a x+b(|x+1|-|x-1|)
$$

and

$\beta=\frac{C_{2}}{C_{1}}, \gamma=\frac{C_{2} R^{2}}{L}, a=m_{1} R, b=\frac{m_{0}-m_{1}}{2} R, x_{1}=\frac{v_{C 1}}{B_{p}}, x_{2}=\frac{v_{C 2}}{B_{p}}, x_{3}=\frac{R i_{L}}{B_{p}}$.

For the fixed values of parameters in a neighborhood of $\gamma=27, \beta=15.6, a=$ $-5 / 7, b=-3 / 14$ and initial conditions $x_{0}=[-0.9,-0.15,1.47]^{T}$, the Chua system presents the so called double scroll chaotic attractors [1]. 
The importance of this circuit stems from its peculiarity of being the only physical system where the presence of chaos has been experimentally established, numerically confirmed and mathematically proven [26]. The circuit can be easily constructed at low cost using standard electronic components and exhibits a rich variety of bifurcations and chaos [27].

Definition 1. Consider a smooth nonlinear system

$$
\dot{x}=f(x, \mathbf{p}), y=h(x),
$$

where $x \in \mathbb{R}^{n}, y \in \mathbb{R}^{m}$ and $\mathbf{p} \in \mathbb{R}^{l}$ with $l<n$ is a constant parameter vector. The state vector $x$ is algebraically observable if it can be uniquely expressed as

$$
x=s\left(y, \ldots, y^{(m)}, \mathbf{p}\right),
$$

for some smooth function s. Moreover, if $\mathbf{p}$ satisfies the following linear relation

$$
s_{1}\left(y, \ldots, y^{(m)}\right)=s_{2}\left(y, \ldots, y^{(m)}\right) \mathbf{p}
$$

where $s_{1}(\cdot)$ and $s_{2}(\cdot)$ are respectively $l \times 1$ and $l \times l$ matrices with $\operatorname{det}\left(s_{2}\left(y, \ldots, y^{(m)}\right)\right) \neq$ 0 , then $\mathbf{p}$ is said to be algebraically linearly identifiable with respect to the output $y$.

System (2) is algebraically observable with respect to the outputs $y_{1}=x_{1}$ and $y_{2}=x_{2}$, since all the system variables can be expressed in terms of $y_{1}, y_{2}$ and their derivatives as

$$
x_{1}=y_{1}, x_{2}=y_{2}, x_{3}=\dot{y}_{2}+y_{2}-y_{1} .
$$

Moreover the system parameters are algebraically linearly identifiable since

$$
\begin{gathered}
\dot{y}_{1}=-\beta(1+a) y_{1}+\beta y_{2}-b \beta z_{1}, \\
\ddot{y}_{2}+\dot{y}_{2}-\dot{y}_{1}=-\gamma y_{2}
\end{gathered}
$$

where $z_{1}=\left|y_{1}+1\right|-\left|y_{1}-1\right|$.

\section{Volterra integral operators algebra}

Let $\mathcal{L}_{\text {loc }}^{2}\left(\mathbb{R}_{\geq 0}\right)$ be the Hilbert space of locally square-integrable functions with domain $\mathbb{R}_{\geq 0}$ and range $\mathbb{R}$. Given a function $f \in \mathcal{L}_{\text {loc }}^{2}\left(\mathbb{R}_{\geq 0}\right)$, its image through the Volterra (linear, integral) operator $V_{K}$ induced by a Hilbert-Schmidt $(\mathcal{H S}$ ) Kernel Function $K(\cdot, \cdot): \mathbb{R} \times \mathbb{R} \rightarrow \mathbb{R}$ is usually denoted by $\left[V_{K} f\right](\cdot)$, and is defined by the inner product:

$$
\left[V_{K} f\right](t) \triangleq \int_{0}^{t} K(t, \tau) f(\tau) d \tau, \quad t \in \mathbb{R}_{\geq 0} .
$$

Any explicit function $f(t): t \rightarrow f_{t} \in \mathbb{R}$, such that $f(\cdot) \in \mathcal{L}_{l o c}^{2}\left(\mathbb{R}_{\geq 0}\right)$ will be addressed to as a signal. Then, given two scalars $a, b \in \mathbb{R}_{\geq 0}$, with $a<b$, let us denote by $f_{[a, b]}(\cdot)$ and $f_{(a, b]}(\cdot)$ the restriction of a signal $f(\cdot)$ to the closed interval $[a, b]$ and to the left open interval $(a, b]$, respectively. Moreover, let $f(t) \in \mathbb{R}^{n}, \forall t \geq 0$ be an $i$-times differentiable vector of signals, we denote by $f^{(i)}$ the vector of the $i$-th order time-derivative signals. Then, we recall the following useful definition: 
Definition 2. Weak (generalized) derivative:

Let $f(\cdot) \in \mathcal{L}_{\text {loc }}^{2}\left(\mathbb{R}_{\geq 0}\right)$. $f^{(1)}(\cdot)$ is said a weak derivative of $f(\cdot)$ if

$$
\int_{0}^{t} f(\tau)\left(\frac{d}{d \tau} \gamma(\tau)\right) d \tau=-\int_{0}^{t} f^{(1)}(\tau) \gamma(\tau) d \tau, \forall t \in \mathbb{R}_{\geq 0}
$$

for all $\gamma \in \mathcal{C}^{\infty}$, with $\gamma(0)=\gamma(t)=0$.

We remark that $f^{(1)}(\cdot)$ is unique up to a set of zero Lebesgue measure, i.e., it is defined almost everywhere. If $f$ is differentiable in the conventional sense, then its weak derivative is identical to its conventional derivative. Traditional rules for the derivation of sum or products of functions also hold for the weak derivative. Given a kernel function $K(\cdot, \cdot)$ in two variables, its $i$-th order weak derivative with respect to the second argument will be denoted as $K^{(i)}, i \in \mathbb{Z}_{\geq 0}$. For the sake of the implementability, a differential form to the operators is devised by applying the Leibniz differentiation rule to the Volterra integral. The transformed signal $\left[V_{K} f\right](t), \forall t \geq 0$, can be obtained as the output of a system described by the scalar integro-differential equation:

$$
\begin{aligned}
& \left\{\begin{array}{c}
\dot{\xi}(t)=K(t, t) f(t)+\int_{0}^{t}\left(\frac{\partial}{\partial t} K(t, \tau)\right) f(\tau) d \tau, t \in \mathbb{R}_{\geq 0} \\
\xi(0)=\xi_{0}=\int_{0}^{t} K(0, \tau) f(\tau) d \tau
\end{array}\right. \\
& {\left[V_{K} f\right](t)=\xi(t), \forall t \in \mathbb{R}_{\geq 0} .}
\end{aligned}
$$

In the following some useful results dealing with the application of Volterra operators to the derivatives of a signal will be recalled.

Lemma 1. Volterra image of a signal's derivative:

For a given $i \geq 0$, consider a signal $f(\cdot) \in \mathcal{L}^{2}\left(\mathbb{R}_{\geq 0}\right)$ that admits a $i$-th weak derivative in $\mathbb{R}_{\geq 0}$ and a kernel function $K(\cdot, \cdot) \in \mathcal{H} \mathcal{S}$ that admits the $i$ th derivative (in the conventional sense) with respect to the second argument, $\forall t \in \mathbb{R}_{\geq 0}$. It holds that:

$$
\begin{aligned}
{\left[V_{K} f^{(i)}\right](t)=} & \sum_{j=0}^{i-1}(-1)^{i-j-1} f^{(j)}(t) K^{(i-j-1)}(t)+ \\
& +\sum_{j=0}^{i-1}(-1)^{i-j} f^{(j)}(0) K^{(i-j-1)}(0)+ \\
& +(-1)^{i}\left[V_{K^{(i)}} f\right](t), \forall t \in \mathbb{R}_{\geq 0}
\end{aligned}
$$

that is, $\left[V_{K} f^{(i)}\right](\cdot)$ is non-anticipative with respect to $f(\cdot)$ and its first $(i-1)$-th derivatives $f^{(1)}(\cdot), \ldots, f^{(i-1)}(\cdot)$.

Lemma 1 allows to identify a class of kernels such that for each derivative $f^{(i)}, i \in\{0, \ldots, n-1\}$, the image signal $\left[V_{K} f^{(i)}\right](t), \forall t>0$ is independent from the initial states $f(0), f^{(1)}(0), \ldots, f^{(i-1)}(0)$, according to the following definition. 
Definition 3. ( $i$-th order non-asymptotic kernel):

Consider a function $K(\cdot, \cdot)$ satisfying the assumptions of Lemma 1; if, in addition, for a given $i \geq 1$, the kernel verifies the condition

$$
K^{(j)}(t, 0)=0, \forall t \in \mathbb{R}_{\geq 0}, \forall j \in\{0, \ldots, i-1\},
$$

then, it is called an $i$-th order non-asymptotic kernel.

Assuming that $K(\cdot, \cdot)$ is an $n$-th order non-asymptotic kernel function, then it holds that:

$$
\begin{aligned}
{\left[V_{K} f^{(i)}\right](t)=} & \sum_{j=0}^{i-1}(-1)^{i-1-j} f^{(j)}(t) K^{(i-j-1)}(t, t)+ \\
& (-1)^{i}\left[V_{K^{(i)}} f\right](t), i \in\{1, \ldots, n-1\} .
\end{aligned}
$$

Considering the case $i=1$, by some trivial manipulation of (9) it follows that

$$
\left[V_{K} f^{(1)}\right](t)=f(t) K(t, t)-\left[V_{K^{(1)}} f\right](t) .
$$

Rearranging (6), for any bounded signal $f(t)$, the transformed signal $\left[V_{K^{(i)}} f\right](t)$ in (9) can be obtained as the output of a linear time-varying dynamic system:

$$
\left\{\begin{array}{l}
\dot{\xi}(t)=K^{(i)}(t, t) f(t)+\int_{0}^{t}\left(\frac{\partial}{\partial t} K^{(i)}(t, \tau)\right) f(\tau) d \tau \\
\xi(t)=\left[V_{K^{(i)}} f\right](t), \forall t \in \mathbb{R}_{\geq 0} .
\end{array}\right.
$$

Let now $K(t, \tau)$ be the following Bivariate Feedthrough Non-asymptotic Kernels $(\mathrm{BF}-\mathrm{NK})$

$$
K(t, \tau)=e^{-\omega_{h}(t-\tau)}\left(1-e^{-\bar{\omega} \tau}\right)^{N},
$$

where $\bar{\omega}, \omega_{h} \in \mathbb{R}_{\geq 0}$ are arbitrarily set constant parameters.

Note for any $i \in\{0,1, \ldots, N\}$, the $i$-th order weak derivative w.r.t. the second argument, $\tau$, is

$$
K^{(i)}(t, \tau)=e^{-\omega_{h} t} \frac{d^{i}}{d \tau^{i}}\left[e^{\omega_{h} \tau}\left(1-e^{-\bar{\omega} \tau}\right)^{N}\right]
$$

with $K^{(i)}(t, \tau)=0, \forall t \in \mathbb{R}_{\geq 0}, i \in\{0,1, \ldots, N\}$.

For the chosen kernel function, for any $i \in\{1, \ldots, N\}$, the partial derivative w.r.t. the first argument $t$ satisfies

$$
\frac{\partial}{\partial t} K^{(i)}(t, \tau)=-\omega_{h} K^{(i)}(t, \tau) .
$$

As a consequence, the first equation in the state space (11) is:

$$
\begin{aligned}
\dot{\xi}(t) & =K^{(i)}(t, t) f(t)+\int_{0}^{t}\left(\frac{\partial}{\partial t} K^{(i)}(t, \tau)\right) f(\tau) d \tau= \\
& =K^{(i)}(t, t) f(t)-\omega_{h} \xi(t), \forall t \in \mathbb{R}_{\geq 0} \\
\xi(0) & =\xi_{0}=0 .
\end{aligned}
$$

Being $K^{(i)}(t, t)$ bounded, it holds that the scalar dynamical system realization of the Volterra operators induced by the proposed kernels is internally stable. 


\section{Kernel-based estimation}

Let us consider differential Eq. (3) with the unknown parameters vector $\theta_{1}=[-\beta(1+a), \beta,-\beta b]^{T}$. Taking the Volterra linear integral operator on both side and considering Kernel functions properties, it follows that

$$
h_{1}(t)^{T} \theta_{1}=s_{1}(t),
$$

where

$$
h_{1}(t)=\left[\left[V_{K} y_{1}\right](t),\left[V_{K} y_{2}\right](t),\left[V_{K} z_{1}\right](t)\right]^{T}
$$

and

$$
s_{1}(t)=K(t, t) y_{1}(t)-\left[V_{K^{(1)}} y_{1}\right](t) .
$$

In order to estimate the parameter $\gamma$, Eq. (4) is considered. In this case, to eliminate the dependence on $\dot{y}_{2}$, two BF-NK functions, namely $K_{1}$ and $K_{2}$, with the same $\bar{\omega}$ and different values of $\omega_{h}\left(\omega_{1}\right.$ and $\left.\omega_{2}\right)$ are used. Letting

$$
\begin{aligned}
s_{2}(t) & =\left[V_{K_{1}^{(2)}} y_{2}\right](t)+\left[V_{K_{1}^{(1)}}\left(y_{1}-y_{2}\right)\right](t)-K_{1}^{(1)}(t, t) y_{2}(t), \\
s_{3}(t) & =\left[V_{K_{2}^{(2)}} y_{2}\right](t)+\left[V_{K_{2}^{(1)}}\left(y_{1}-y_{2}\right)\right](t)-K_{2}^{(1)}(t, t) y_{2}(t), \\
h_{2}(t) & =\left[V_{K_{2}} y_{2}\right](t)-\left[V_{K_{1}} y_{2}\right](t),
\end{aligned}
$$

the algebraic equation

$$
h_{2}(t) \gamma=s_{2}(t)-s_{3}(t)
$$

is obtained.

In the following, for the sake of brevity, the estimation procedure will be explained for the parameters vector $\theta_{1}$ case only. The same approach can be easily adapted to estimate the parameter $\gamma$.

From Eq. (15) it follows that the parameters vector $\theta_{1}$ verifies the structural constraint

$$
H(t) \theta_{1}-S(t)=0,
$$

where

$$
H(t)=h_{1}(t) h_{1}(t)^{T}, \quad S(t)=h_{1}(t) s_{1}(t) .
$$

Let $H_{f}(t)$ and $S_{f}(t)$ be two filtered versions of the matrices $H(t)$ and $S(t)$ :

$$
\left\{\begin{array}{l}
\dot{H}_{f}(t)=-\lambda H_{f}(t)+H(t) \\
\dot{S}_{f}(t)=-\lambda S_{f}(t)+S(t)
\end{array}\right.
$$

with $H_{f}(0)=0 \in \mathbb{R}^{3 \times 3}$ and $S_{f}(0)=0 \in \mathbb{R}^{3}$, then the following structural constraint is verified too

$$
H_{f}(t) \theta_{1}-S_{f}(t)=0 .
$$

For an estimate $\hat{\theta}_{1}(t)$ (possibly $\hat{\theta}_{1}(t) \neq \theta$ ), the above constraint is not exactly matched, and in general a non-zero residual $\tilde{R}_{f}(t)$ is introduced:

$$
\tilde{R}_{f}(t)=H_{f}(t) \hat{\theta}_{1}(t)-S_{f}(t), \quad \dot{\tilde{R}}_{f}(t)=H_{f}(t) \dot{\hat{\theta}}_{1}(t)+\dot{H}_{f}(t) \hat{\theta}_{1}(t)-\dot{S}_{f}(t) .
$$


In order to make $\left\|\tilde{R}_{f}(t)\right\| \rightarrow 0$ in finite time the following sliding-mode type adaptation law can be used:

$$
\dot{\hat{\theta}}_{1}(t)=\left\{\begin{array}{l}
H_{f}(t)^{-1}\left[-\mu \operatorname{sign}\left(\tilde{R}_{f}(t)\right) \sqrt{\left|\tilde{R}_{f}(t)\right|}-\dot{H}_{f}(t) \hat{\theta}_{1}(t)+\dot{S}_{f}(t)\right], \\
\quad \text { if }\left|\operatorname{det}\left(H_{f}(t)\right)\right| \geq \delta, \\
\quad \text { if }\left|\operatorname{det}\left(H_{f}(t)\right)\right|<\delta,
\end{array}\right.
$$

where $\delta>0$ is a user-defined threshold constant.

Note that the proposed adaptation law uses the derivatives of $H_{f}(t)$ and $S_{f}(t)$ instead of $\dot{H}(t)$ and $\dot{S}(t)$ because the above signals are not available.

Remark 1. In summary the algorithm requires, $\forall t \geq 0$, the implementation of Eqs. (16)-(17) and (20)-(23). In particular, as far as the computational effort is concerned, Eqs. (16)-(17) require the computation of the Volterra image of signals derivatives and, according to the previous theoretical results, it translates into the application of a first order filter; Eq. (21) implies the computation of filtered versions of matrices $H$ and $S$, i.e. the application of a first order filter at each matrix element. Finally in Eq. (22) the inverse of $H_{f}(t)$ is required.

\subsection{Finite-time convergence in noise-free scenario}

In this Section, the convergence properties of the proposed estimator in absence of external perturbations is addressed.

Assumption 1. (Persistency of Excitation): The vector $h_{1}(t)$ is persistently exciting (PE) in $\mathbb{R}^{3}$ in the sense that exsist constants $r>0$ and $T>0$ such that $\forall t \geq 0$

$$
\int_{t-T}^{t} h_{1}(\tau) h_{1}^{T}(\tau) d \tau \geq r I>0
$$

Lemma 2. Given a vector $x=\left[x_{1}, \ldots, x_{n}\right] \in \mathbb{R}^{n}$ and $p \in(0,1)$, it holds that

$$
\sum_{i=1}^{n}\left|x_{i}\right|^{p} \geq\left(\sum_{i=1}^{n}\left|x_{i}\right|\right)^{p}
$$

Lemma 3. Deadbeat estimation algorithm convergence time:

Let $V(t)>0 \forall t \geq t_{0}$, and let

$$
\dot{V}(t) \leq-c V^{\eta}(t), \forall t \geq t_{0},
$$

with $c>0$ and $\eta \in(0,1)$; then

$$
\begin{cases}V^{1-\eta}(t) \leq V^{1-\eta}\left(t_{0}\right)-c(1-\eta)\left(t-t_{0}\right), & t \in\left[t_{0}, t_{1}\right] \\ V(t)=0, & \forall t \geq t_{1},\end{cases}
$$

with

$$
t_{1}=t_{0}+\frac{V^{1-\eta}\left(t_{0}\right)}{c(1-\eta)}
$$


Proof. Given the following differential equation

$$
\left\{\begin{array}{l}
\dot{x}(t)=-c x^{\eta}(t) \\
x\left(t_{0}\right)=V\left(t_{0}\right),
\end{array}\right.
$$

its solution is

$$
x^{1-\eta}(t)=-c(1-\eta)\left(t-t_{0}\right)+x^{1-\eta}\left(t_{0}\right),
$$

the derivative of which is

$$
\frac{d}{d t} x^{1-\eta}(t)=-c(1-\eta)<0 .
$$

Then the function $x^{1-\eta}(t)$ is monotonically decreasing; as a consequence

$$
V^{1-\eta}(t) \leq V^{1-\eta}\left(t_{0}\right)-c(1-\eta)\left(t-t_{0}\right), \forall t_{0} \leq t \leq t_{1} .
$$

Moreover $V^{1-\eta}\left(t_{1}\right)=0$ where

$$
t_{1}=t_{0}+\frac{V^{1-\eta}\left(t_{0}\right)}{c(1-\eta)}
$$

Since $V^{1-\eta}(t)$ is monotonically decreasing, $V(t)$ is monotonically decreasing too, and since $V(t) \geq 0$ then

$$
V(t)=0, \forall t \geq t_{1}
$$

Theorem 1. If Assumption 1 holds, given the noise-free signals $y_{1}(t), y_{2}(t), z_{1}(t)$, the estimated parameters vector $\theta_{1}(t)$ governed by the adaptation law (23) converges to the true value $\theta_{1}$ in finite-time.

Proof. Let define the following Lyapunow function

$$
V(t)=\frac{1}{2} \tilde{R}_{f}^{T}(t) \tilde{R}_{f}(t),
$$

the derivative of which is

$$
\dot{V}(t)=\tilde{R}_{f}^{T}(t) \dot{\tilde{R}}_{f}(t)
$$

Substituting (22) and (23) into (27) it follows that

$$
\dot{V}(t)=-\mu \sum_{i=1}^{3}\left|\tilde{R}_{f, i}(t)\right|^{\frac{3}{2}}
$$

where $\tilde{R}_{f, i}(t)$ is the $i$-th component of $\tilde{R}_{f}(t)$.

$\dot{V}(t)$ can now be written in terms of $V(t)$. Starting from (28), one obtains that

$$
\begin{aligned}
\dot{V}(t) & =-\mu \sum_{i=1}^{3}\left|\tilde{R}_{f, i}(t)\right|^{\frac{3}{2}}=-\mu \sum_{i=1}^{3}\left(\left|\tilde{R}_{f, i}(t)\right|^{2}\right)^{\frac{3}{4}}= \\
& =-2^{\frac{3}{4}} \mu \sum_{i=1}^{3}\left(\frac{1}{2}\left|\tilde{R}_{f, i}(t)\right|^{2}\right)^{\frac{3}{4}} .
\end{aligned}
$$


Applying Lemma 2, the Eq. (29) becomes

$$
\dot{V}(t)=-2^{\frac{3}{4}} \mu \sum_{i=1}^{3}\left(\frac{1}{2}\left|\tilde{R}_{f, i}(t)\right|^{2}\right)^{\frac{3}{4}}<-2^{\frac{3}{4}} \mu\left(\frac{1}{2} \sum_{i=1}^{3}\left|\tilde{R}_{f, i}(t)\right|^{2}\right)^{\frac{3}{4}} .
$$

Recalling now (26),

$$
\dot{V}(t)<-2^{\frac{3}{4}} \mu V(t)^{\frac{3}{4}}
$$

and thus the proof follows. The convergence time can be easily obtained as shown in Lemma 3.

\subsection{Robustness Analysis}

In this Section, the stability properties of the proposed estimation technique, in the case of noisy measurements, are studied.

Let assume that

$$
\begin{aligned}
& \bar{y}_{1}(t)=y_{1}(t)+d_{y, 1}(t) \\
& \bar{y}_{2}(t)=y_{2}(t)+d_{y, 2}(t) \\
& \bar{z}_{1}(t)=z_{1}(t)+d_{z, 1}(t)
\end{aligned}
$$

where $\left|d_{y, 1}(t)\right| \leq \bar{d}_{y},\left|d_{y, 2}(t)\right| \leq \bar{d}_{y}$ and $\left|d_{z, 1}(t)\right| \leq \bar{d}_{z}$.

Starting from Eq. (19), the perturbation on the measurements can be seen as a noisy input to Volterra integral operator shown in (14) and due to the internal stability of this operator, the output to this noisy bounded input will be bounded too. As a consequence the signals $S(t)$ and $H(t)$ become

$$
\begin{aligned}
& \bar{S}(t)=S(t)+\Delta S(t) \\
& \bar{H}(t)=\bar{h}_{1}(t) \bar{h}_{1}^{T}(t) \quad=H(t)+\Delta H(t)
\end{aligned}
$$

where $\Delta S(t)$ and $\Delta H(t)$ are related to the measurements noises and are bounded too.

Let now assume that $\bar{h}_{1}(t)$ is persistently exciting in $\mathbb{R}^{3}$; at this point the filtered signals

$$
\begin{cases}\dot{\bar{H}}_{f}(t) & =-\lambda \bar{H}_{f}(t)+\bar{H}(t) \\ \dot{\bar{S}}_{f}(t) & =-\lambda \bar{S}_{f}(t)+\bar{S}(t)\end{cases}
$$

can be used to define the following sliding-mode type adaptation law

$$
\dot{\hat{\theta}}_{1}(t)=\bar{H}_{f}^{-1}(t)\left(-\mu \operatorname{sign}\left(\overline{\tilde{R}}_{f}(t)\right) \sqrt{\left|\tilde{\tilde{R}}_{f}(t)\right|}+\dot{\bar{S}}_{f}(t)-\dot{\bar{H}}_{f}(t) \hat{\theta}_{1}(t)\right) .
$$

The estimation $\hat{\theta}_{1}(t)$ then converges in the bounded region

$$
\hat{\theta}_{1}(t) \in\left[\inf _{0 \leq \tau \leq t}\left(\bar{H}_{f}(\tau)\right)^{-1} \bar{S}_{f}(\tau), \sup _{0 \leq \tau \leq t}\left(\bar{H}_{f}(\tau)\right)^{-1} \bar{S}_{f}(\tau)\right] .
$$

In conclusion, the estimation error $\hat{\theta}_{1}(t)-\theta_{1}$ is bounded with respect to bounded disturbances. 


\section{A numerical example}

To evaluate the performance of the proposed parameters estimation technique in both noisy and noise-free scenarios,numerical tests have been performed starting from the initial conditions $x_{1}(0)=-0.9, x_{2}(0)=-0.15, x_{3}(0)=1.47$ and with the following parameters to be obtained:

$$
\gamma=27, \quad \beta=15.6, \quad a=-5 / 7, \quad b=-3 / 14 .
$$

The proposed Kernel based estimation technique (denoted as $\mathbf{K B M}$ ) has been performed using the parameters shown in Tab. 1.

\begin{tabular}{l|l}
\hline Parameter & Value \\
\hline$\omega_{h}$ & 0.5 \\
$\bar{\omega}$ & 0.1 \\
$\omega_{1}$ & 0.5 \\
$\omega_{2}$ & 0.8 \\
$\lambda$ & 0.05 \\
$\mu$ & 0.001 \\
$\delta$ & $10^{-4}$ for parameters $a, b$ and $\beta$ \\
& $10^{-6}$ for parameter $\gamma$ \\
\hline
\end{tabular}

Table 1: KBM parameters values

and it has been contrasted with the technique proposed in [20], hereafter denoted as linear method $(\mathbf{L M})$, where a linear system of equations is built starting from the output $y(t)=x_{1}(t)$ and assuming to have knowledge about the parameters $a$ and $b$. Solving the above systems of equations, information about the initial conditions on $x_{2}$ and $x_{3}$ is inferred along with an estimation of the missing parameters.

In the noisy case, the state variables have been assumed to be corrupted by a uniformly distributed noise in $[-0.05,0.05]$. The resulting Chua system evolution is shown in Fig. 2.

Figs. 3 and 4 show the obtained estimations in noise-free and noisy scenarios respectively: for the parameters $\beta$ and $\gamma$, the results obtained using both the methods are shown; for the remaining coefficients, only the finite time estimations are depicted.

According to these figures, $\mathbf{K B M}$ activated at around $2 \mathrm{~s}$ for the parameters $a, b, \beta$ and at around $6 \mathrm{~s}$ for $\gamma$ and it converges at $6 \mathrm{~s}$ and $12 \mathrm{~s}$ respectively. In the noisy case it performs a smoother estimation for the unknown parameters and the estimation error is bounded and it shows lower fluctuations around the steady state value w.r.t. LM.

As a main drawback, the proposed method requires information about both voltages on the capacitors, while the method described in [20] needs only one of them. However, the latter method assumes to have partial knowledge about the Chua system parameters. Moreover, no assurance is given about robustness of the linear method w.r.t. noise. In particular, to increase robustness, the linear set of equations has to be solved in a least mean square way using a big number of measurements and then requiring high spatial cost and computational effort. 


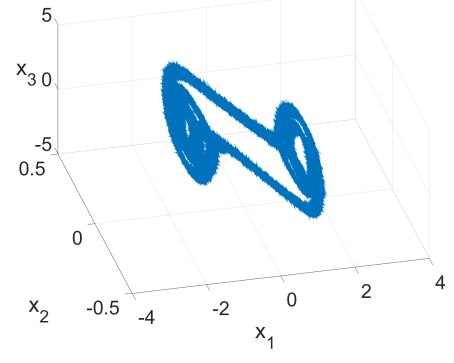

(a) Evolution in the $x_{1}, x_{2}, x_{3}$ space

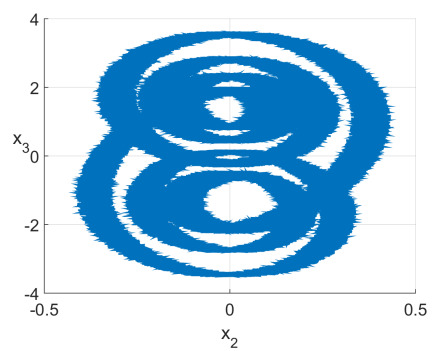

(c) Evolution in the $x_{2}, x_{3}$ plane

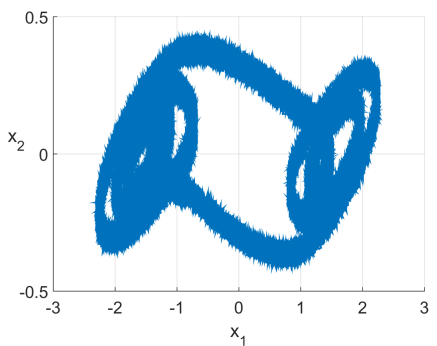

(b) Evolution in the $x_{1}, x_{2}$ plane

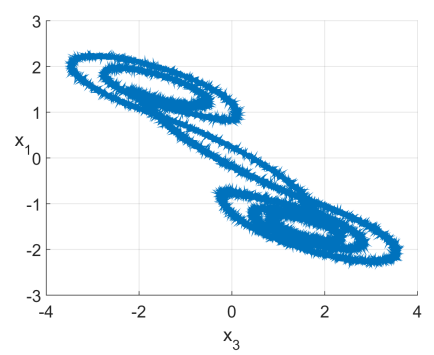

(d) Evolution in the $x_{3}, x_{1}$ plane

Figure 2: Double scroll.

\section{Conclusions}

In this paper a novel parameters estimation technique for the Chua system has been proposed. First of all, the Chua system has been analyzed showing that it is algebraically observable w.r.t. the voltages on the capacitors. Assuming to have knowledge about the above signals, a finite time parameters estimation method has been proposed using the Volterra integral operator properties. Moreover, it has been proven that the parameters estimation error is bounded if the system is corrupted by an additive bounded noise. The resulting technique has been tested in numerical simulations showing its effectiveness in providing the system parameters in finite time.

\section{References}

[1] G. Fedele, A. Ferrise, F. Chiaravalloti, Uncertain master-slave synchronization with implicit minimum saturation level, Applied Mathematical Modelling, 40(2), 1193-1198 (2016).

[2] X. Wu, Z. Wang, Estimating parameters of chaotic systems under noiseinduced synchronization, Chaos Solitons \& Fractals, 39, 689-696 (2009).

[3] A. Isidori, Nonlinear Control Systems, 2nd Edition, Springer-Verlag, Berlin, (1989).

[4] H. Huijberts, H. Nijmeijer, R. Willems, System Identification in Communication with Chaotic Systems, IEEE Transactions on Circuits and Systems I: Fundamental Theory and Applications, 47, 800-808 (2000). 


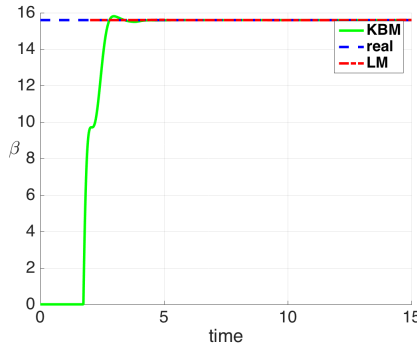

(a) Parameter $\beta$ estimation results.

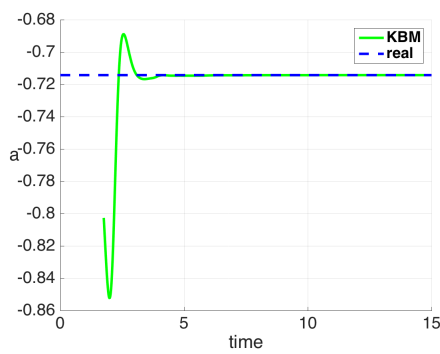

(c) Parameter $a$ estimation results

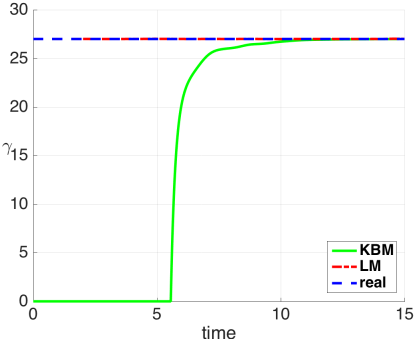

(b) Parameter $\gamma$ estimation results.

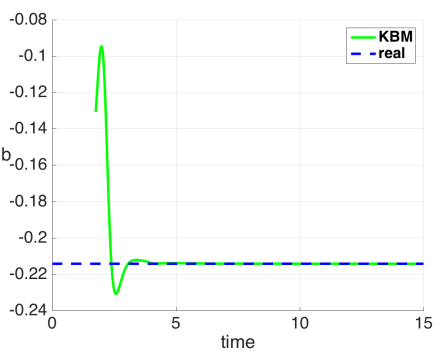

(d) Parameter $b$ estimation results.

Figure 3: Estimation in noise-free scenario

[5] F. Takens, Detecting strange attractors in turbulence, in D.A. Rand, L.S. Young (Eds.), Dynamical Systems and Turbulence, Springer-Verlag, Berlin, 366-381 (1981).

[6] U. Parlitz, R. Zöller, J. Holzfuss, W. Lauterborn, Reconstructing physical variables and parameters from dynamical systems, International Journal of Bifurcation and Chaos, 4(6), 1715-1719 (1994).

[7] T. Stojanovski, U. Parlitz, L. Kocarev, R. Harris, Exploiting Delay Reconstruction for Chaos Synchronization, Phys. Lett. A, 233, 355-360 (1997).

[8] U. Parlitz, Identification of true and spurious Lyapunov exponents from time series, International Journal of Bifurcation and Chaos, 2(1), 155-165 (1992).

[9] A.H. Nayfeh, D.T. Mook, Non-Linear Oscillations, Wiley, Chichester (1979).

[10] X. Wu, Z. Wang, Estimating parameters of chaotic systems synchronized by external driving signal, Chaos Solitons \& Fractals, 33, 588-594 (2007).

[11] X. Wu, Z. Wang, Estimating parameters of chaotic systems under noiseinduced synchronization, Chaos, Solitons \& Fractals, 39(2), 689-696 (2009).

[12] W. Yu, High-gain Observer for chaotic synchronization and secure communication, International Journal of Bifurcation and Chaos, 18, 487-500 (2005).

[13] M. Itoh, C.W. Chai, L.O. Chua, Communication Systems via Chaotic Signals from a Reconstruction Viewpoint, International Journal of Bifurcation and Chaos, 7, 275-286 (1997). 


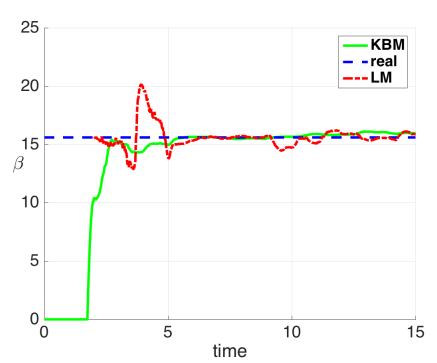

(a) Parameter $\beta$ estimation results.

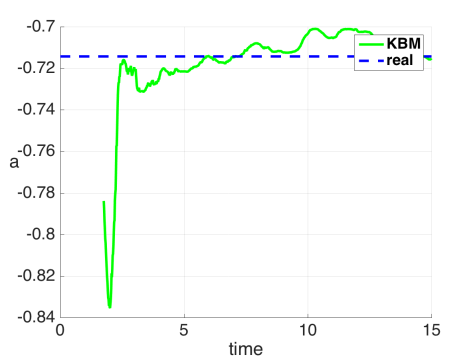

(c) Parameter $a$ estimation results

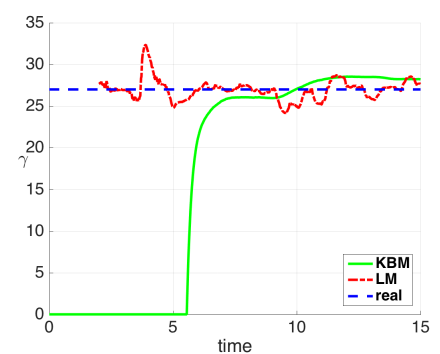

(b) Parameter $\gamma$ estimation results.

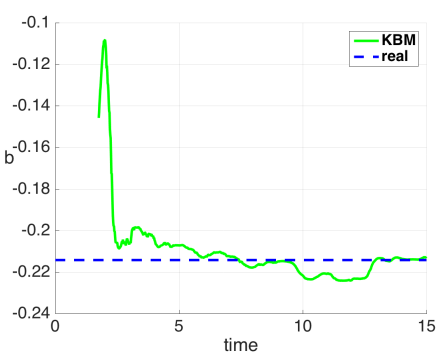

(d) Parameter $b$ estimation results.

Figure 4: Estimation in noisy scenario

[14] M. Hasler, Synchronization of chaotic systems and transmission of information, International Journal Bifurcation and Chaos, 9(3), 647-659 (1999).

[15] L.M. Pecora, T.L. Carroll, Synchronization in chaotic system, Physical Review Letters, 64, 821-824 (1990).

[16] T. Ushio, Synthesis of synchronized chaotic system based on observers, International Journal of Bifurcation and Chaos, 9(3), 541-546 (1999).

[17] M.S. Suárez-Castañón , C. Aguilar-Ibáñez, R. Barrón-Fernández , On recovering the parameters and velocity state of the Duffin's oscillator, Physics Letters A, 308, 47-53 (2003).

[18] C. Aguilar-Ibáñez, On recovering the parametric model of the Chua system via a gradient algorithm, Revista Mexicana de Física, 53(6), 436-440 (2007).

[19] G.L. Baker, J.P. Gollub, and J.A. Blackburn, Inverting Chaos: Extracting System Parameters from Experimental Data, Chaos, 6, 528-553 (1996).

[20] M.S. Suarez-Castañon, C. Aguilar-Ibáñez, and F. Flores-Ando, Reconstructing the states and parameters of Chua's system based on successive integrations of the output, Physics Letters A, 317(3-4), 265-274 (2003).

[21] P. Li, G. Pin, T. Parisini, G. Fedele, Deadbeat Source Localization from Range-only Measurements: a Robust Kernel-based Approach, American Control Conference, 6-8 July, 2729-2734 (2016). 
[22] P. Li, G. Fedele, G. Pin, T. Parisini, Kernel-Based Deadbeat Parametric Estimation of Bias-Affected Damped Sinusoidal Signals, European Control Conference, 15-17 July, 479-484 (2015).

[23] G. Pin, A. Assalone, M. Lovera and T. Parisini, Non-Asymptotic KernelBased Parametric Estimation of Continuous-Time Linear Systems, IEEE Transactions on Automatic Control, 61(2), 360-373 (2016).

[24] F. Chiaravalloti, L. D'Alfonso, G. D'Aquila, G. Fedele, T. Parisini, G. Pin, Finite-time parameters estimation of the Chua system, AIP Conference Proceedings 1776, 090024 (2016); doi: 10.1063/1.4965388.

[25] T. Matsumoto, L.O. Chua, M. Komuro, The Double Scroll, IEEE Trans. Circuits Syst., vol. CAS-32(8), 798-818, 1985.

[26] L. Pivka, Ladislav, C.W. Wu, A. Huang, Chua's oscillator: a compendium of chaotic phenomena, Journal of the Franklin Institute 331(6), 705-741 (1994).

[27] E. Bilotta, P. Pantano, A gallery of Chua attractors. Vol. 61. World Scientific, 2008. 